\title{
Cuba: cambiando el rumbo
}

\author{
Judith Hernández \\ Guillermo Foladori
}

Entre 2008 y 2011, el gobierno cubano decretó una serie de medidas con el propósito de cambiar el rumbo de la economía y la sociedad cubanas. Este artículo explica los problemas que pretenden corregir estas medidas $y$, al hacerlo, muestra la contradicción que generó el desarrollo del socialismo cubano y que obligó a modificar el rumbo del desarrollo.

\section{PRINCIPALES CARACTERÍSTICAS DE LA ECONOMÍA CUBANA}

L a planificación y, por tanto, el control del Estado sobre la economía, fue la principal característica del sistema económico cubano posrevolucionario. El mercado fue sustituido por mecanismos de distribución estatizados que controlaron la ley del valor, eliminando el dominio del capital, tanto nacional como extranjero. La segunda característica fue la integración - hasta el derrumbe en 1989- de la economía cubana a la división internacional socialista del trabajo, centralizada en la antigua Unión de Repúblicas Soviéticas Socialistas (URSS). Esto implicó un fuerte subsidio, una especialización que hacía dependiente a Cuba de la producción industrial de la URSS y una pérdida de autonomía alimenticia en varios rubros. La tercera característica fue el desarrollo de la economía en el marco de un feroz bloqueo económico por Estados Unidos. El modelo de desarrollo orientado a satisfacer las necesidades cristalizó, en pocas décadas, en una fuerte reducción de la pobreza y las desigualda- 
des sociales a partir de una equitativa distribución del ingreso. La isla mostró un amplio desarrollo social.

A principios de los noventa sobreviene una profunda crisis estructural que abarca todas las esferas de la sociedad y pone a prueba la sobrevivencia de la Revolución. El país pierde ipso facto al sistema socialista mundial, base del modelo de acumulación y consumo que perduró por tres décadas. Se declara el «Periodo Especial en Tiempo de Paz» con el objetivo de hacer uso racional de los pocos recursos con que contaba la isla y, a la vez, mantener los logros sociales alcanzados.

En este panorama, el reforzamiento del bloqueo económico por

las estrategias de desarrollo. Surge el Modelo Económico Heterogéneo o Mixto de la Transición (1990-2011). ${ }^{1}$ Esta estrategia de desarrollo adoptó una reforma económica guiada por el principio de mantener las conquistas sociales alcanzadas en las etapas anteriores y retomar la construcción del socialismo una vez creadas las condiciones.

Al contrario de lo esperado, las reformas no lograron mecanismos endógenos de acumulación. Por ello, y por deficiencias en el uso de las políticas de racionamiento puestas en práctica después del triunfo revolucionario, se mantuvieron las limitaciones en el consumo, bajos ingresos y subempleo derivado de políticas sociales de pleno empleo, la restricción a la movilidad de las personas y el carácter restrictivo y político de la migración — dada por el diferendo entre Estados Unidos y Cuba-, los bajos niveles de productividad, la dependencia de la economía hacia sectores externos, el amplio sistema de políticas sociales sobre la base de compensaciones externas y un fuerte control social a través de la política.

A partir de 2008 se lleva a cabo un nuevo conjunto de medidas para forzar a una reorientación económica. Antes de analizar este cambio de rumbo, es necesario entender qué contradicciones generó el desarrollo del socialismo cubano.

\section{LA DENSIDAD DE POBLACIÓN}

El concepto de densidad de población se utiliza en demografía para hacer referencia a la cantidad de población por unidad de superficie. En economía política el concepto es más amplio, y no mide la población por unidad de superficie, sino que evalúa la cantidad de población necesaria para desarrollar actividades económicas dado un nivel de desarrollo de las fuerzas productivas en un espacio determinado. James Mill ${ }^{2}$ argumentaba que una determinada densidad de población era conveniente, tanto para el trato social como para combinar las fuerzas que aumentan la productividad del trabajo. Y Marx, retomando las palabras de Mill, argumentaba que en el concepto de densidad de población había que considerar no sólo las condiciones productivas sino los medios de transporte y comunicación, que facilitan su integración a los procesos productivos, por ello el sur de

Estados Unidos podía estar más densamente poblado que India.

En las sociedades capitalistas el mercado genera un permanente acicate al aumento de la productividad del trabajo. El capitalismo resuelve la proporción entre población y necesidades productivas (densidad de población) generando una masa de fuerza de trabajo excedente, que el capital atrae y rechaza (inclusive de manera transfronteriza) según los ciclos productivos y económicos, creando así una amplia zona de pobreza y desigualdad social sin protección. Pero Cuba no es una economía capitalista. Y una de las características más sobresalientes de su economía era la baja tasa de desempleo y la amplia cobertura de los beneficios sociales. Sin el ejército de reserva capitalista como regulador de la densidad de población y sin el acicate del mercado para aumentar la productividad del trabajo, Cuba se enfrentó a la combinación de los siguientes factores: 1) ninguna elasticidad en la oferta/demanda de empleo y altos índices de ineficiencia; 2) una parte importante del empleo ocupado en tareas improductivas (e.g. administración pública, salud y educación), y 3) elevado nivel de conciencia que, sumado a la crisis económica, provocó una fuerte emigración y una drástica disminución de las tasas de fecundidad. El resultado fue una dinámica poblacional que convirtió en insustentable el desarrollo económico.

El cuadro 1 muestra los resultados del amplio sistema de políticas sociales establecido por el gobierno cubano, que superó los problemas de extrema pobreza, escasa educación y de salud y cultura restringida a unos pocos. Un amplio grupo de programas contribuyeron a lograr altos niveles de desarrollo social de la población, a saber: los programas de salud gratuitos dirigidos a la atención primaria, materno-infantil, puericultura, tercera edad; los programas educativos con acceso general y gratuito de la población a los niveles primarios, secundarios, preuniversitarios y universitarios; los programas de asistencia social. 
Evolución de indicadores sociales seleccionados.

\begin{tabular}{|c|c|c|c|}
\hline $\begin{array}{c}\text { INDICADORES } \\
\text { Tasa de mortalidad infantil (por mil nacidos } \\
\text { vivos) }\end{array}$ & 1960 & 1980 & 1990 \\
\hline Habitantes por médico & 19.6 & 10.7 \\
\hline Esperanza de vida al nacer (años) & 1076 & 635 & 275 \\
\hline Analfabetos (\%) & 57 & 72.5 & 74 \\
\hline Envejecimiento de la población (\% > 60 años) & 8.0 & 10.8 & 0.4 \\
\hline Desempleo (\%) & 23.7 & $6.0 \%$ & 11.8 \\
\hline Número de escuelas (u) & 7676 & 12908 & 12511 \\
\hline Centros de Educación Superior & 3 & 28 & 35 \\
\hline
\end{tabular}

Fuente: Elaboración propia a partir de ONE (varios años), Anuario Estadístico de Cuba; Raúl Hernández Castellón, El envejecimiento de la Población en Cuba, CEDEM, Universidad de la Habana, 1994; Elena C. Álvarez González, Cuba, un modelo de desarrollo con justicia social, 1998; Anicia García Álvares y Betsy Anaya Cruz, Relación entre desarrollo social y económico, en Omar Everleny Pérez Villanueva (compilador), Cincuenta años de desarrollo económico y social en Cuba, Editorial Ciencias Sociales, La Habana, 2010. *Se refiere a 1989. *"Se refiere a 1997.

La gran limitación en el consumo individual, contrastado a la imagen de amplio consumo de Estados Unidos, propició la emigración basada en la expectativa de mejoras económicas. Esta emigración repercutió sobre la dinámica demográfica extremando todos sus indicadores. En el gráfico 1 se observa la correspondencia entre la Tasa Anual de Crecimiento (TAC) y la Tasa de Saldo Migratorio Externo (TSME). La TAC sufre varias caídas: la primera en 1980, resultado de la ola migratoria de «los marielitos» con pérdidas de 6.2 efectivos por cada mil habitantes; en 1994 cae a 1.60 por mil habitantes, correspondiendo con la «crisis de los balseros». En los siguientes años el indicador apenas se recupera. Pero nótese que es en 2005-2006 cuando la TAC tiene un punto de inflexión, dando paso a la caída a números negativo $(2006,-0.40)$. Obsérvese que en 2006, a pesar que el crecimiento natural se comportó sobre tres por mil, la TAC experimenta una pérdida absoluta de 4,793 habitantes, que se debe a la sangría que provoca la emigración.

La isla presenta decrecimiento poblacional. Éste no sólo es resultado de los altos niveles de migración sino también de la fuerte contracción de la tasa de fecundidad. La Tasa Global de Fecundidad (TGF) se ubicaba en 1980 en 1.67 hijos por mujer, alcanzando su nivel más alto en 1985, con 1.91 hijos por mujer. Con la declaración del «Periodo Especial» comenzó un drástico descenso, hasta ubicarla en su nivel más bajo. En 2006 alcanza una tasa de 1.39 hijos por mujer, recuperándose nuevamente hasta 1.78 en 2011, pero sin llegar a niveles de reemplazo poblacional.

La combinación de alta migración y baja fecundidad es explosiva para la isla, comprometiendo su futuro desarrollo. El resultado es una dinámica demográfica que combina una disminución de la Población Económicamente Activa (PEA), pocas posibilidades de reposición de la fuerza laboral y un alto envejecimiento. El gráfico 2 muestra la dinámica demográfica de los diferentes grupos etarios. El grupo de edad 
GRÁFICO 1

Evolución de la Tasa del Saldo Migratorio Externo (TSME) y la Tasa Anual Crecimiento (TAC).

POR MIL HAB.

15

10

5

\begin{tabular}{l}
\hline \\
\hline
\end{tabular}

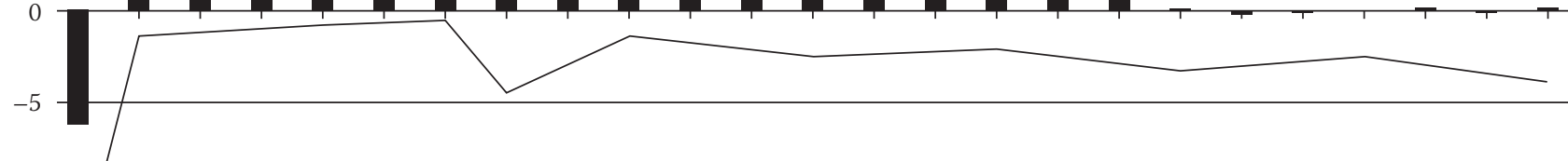

$-10$

$-15$

$-20$

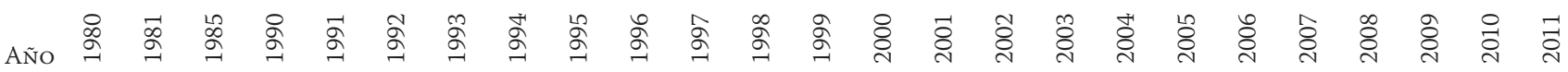

TAC - TSME

Fuente: elaboración propia a partir de los datos del ONE

(Oficina Nacional de Estadística de la República de Cuba) (varios años).

GRÁFICO 2

Dinámica de la población por grupo de edad. Pre-laboral (0-14 años), Laboral (pea) y Post-laboral (+ 60 años).

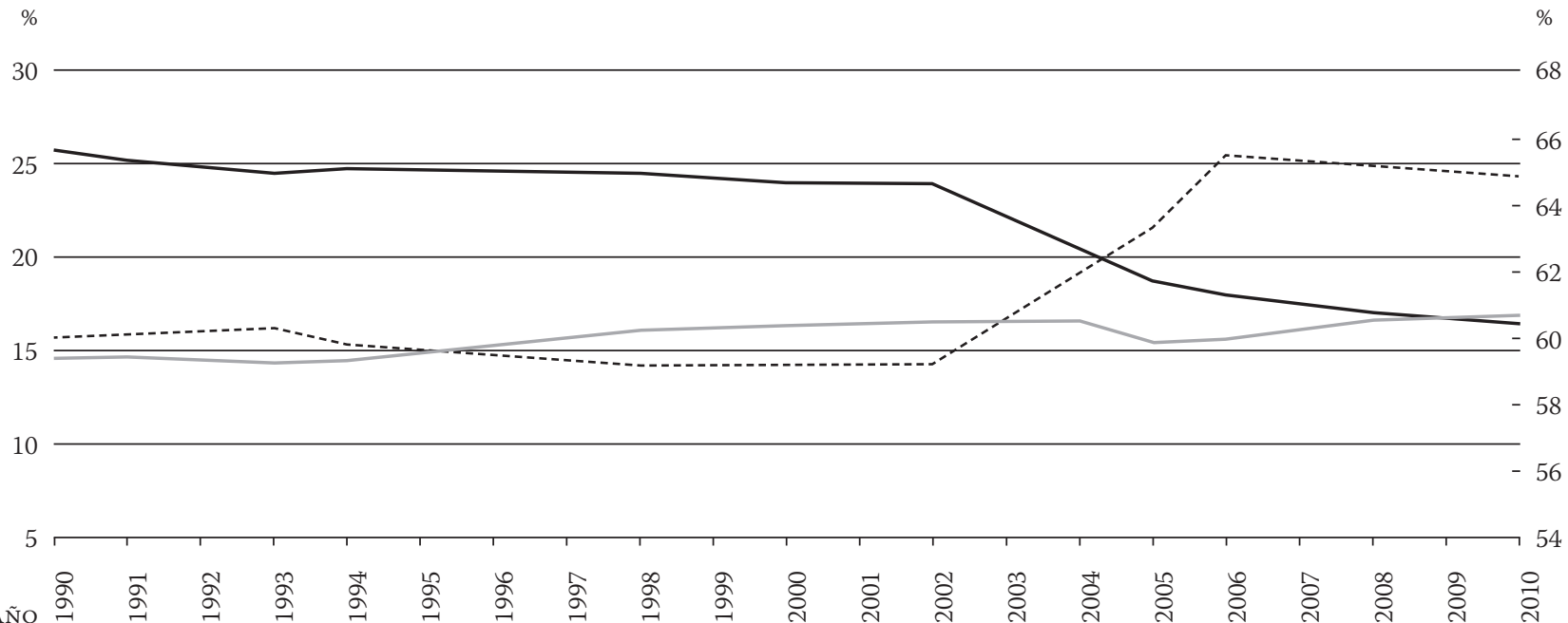

- \% 0-14 años — \% $\% 60$ años --- \% PEA

Fuente: elaboración propia a partir de los datos del ONE

(Oficina Nacional de Estadística República de Cuba) (varios años). 
de 0-14 años representa la población joven y, por tanto, el grupo encargado de reponer la fuerza laboral activa. El grupo mayor de 60 años ilustra el envejecimiento. Ambos grupos no realizan aportes económicos; son considerados dependientes de la PEA que es la fuerza productiva del país impulsora del desarrollo económico.

La población joven (0-14 años; línea oscura) tiende a la disminución. En 1990 este grupo representaba $26 \%$ de la población, diez años después $23 \%$ y al término de 2010 sólo 17\% del total de la población cubana. Por su parte, la población mayor de 60 años (línea clara) muestra un comportamiento contrario, su tendencia en el tiempo es al incremento. En 1990 era de 14\%, en 2010 de 18\%, que es un porcentaje aún mayor que el grupo de edad de 0-14 años y así una situación muy preocupante para la economía cubana. La PEA (línea discontinua) mantiene un comportamiento irregular: en el periodo 1990-1993 aumenta ligeramente, pasando de $54 \%$ a $61 \%$. A partir de 1994, con la ocurrencia de la «crisis de los balseros», disminuye hasta llegar a su nivel más bajo en 2002 al constituir 59\% de la población. En 2006 aumenta a su nivel más alto (65\%) y nuevamente comienza el descenso.

El resultado general es que el desarrollo económico socialista no consiguió articular capacidades productivas y trabajo. Es claro que las condiciones externas fueron determinantes, pero esto levanta la pregunta de si es viable el socialismo en un país aislado sujeto a bloqueo económico. Para el quiebre de los siglos la economía cubana se había vuelto inviable.

\section{LAS REFORMAS}

A partir de 2008 se lleva a cabo un conjunto de medidas. Una de las primeras fue dirigida a atenuar las tendencias demográficas que reducían la PEA. Se promulga en 2008 la ley no. 105/08: Ley de Seguridad Social (ss). La ley propone la extensión a cinco años, para ambos sexos, de la vida laboral y de cotización para acceder a la ss. Esto presupone que las mujeres serán jubiladas a los 60 años y los hombres a los 65, incluyendo 30 años de servicio en ambos casos. Además, la reincorporación a la vida laboral de los jubilados que lo deseen sin renuncia a la pensión. También existen nuevos beneficios por la modificación del cálculo de las pensiones, más acorde con los aportes de los trabajadores, y una contribución especial a la seguridad social de todos los trabajadores e incorporación a un régimen especial integrador de la ss para los trabajadores por cuenta propia. La ley no sólo busca incrementar la PEA sino también extender la vida útil del personal calificado, lo cual es urgente debido a la fuerte migración de profesionales.

Mas esta ley no resuelve el acuciante problema de la disponibilidad de recursos humanos ni la disminución de la alta dependencia de los no trabajadores sobre los trabajadores. La relativa salud de los ancianos y la conservación de sus capacidades físicas y mentales, o sea, el hecho de aumentar su esperanza de vida, no es factor suficiente para la prolongación de su vida productiva, además de que obliga a reformas en el sistema de salud para acompañar la permanencia en el trabajo (geriatría y gerontología) que pueden ser más costosas.

Otra medida importante se anuncia en 2009 con el decreto-ley no. 268 modificaciones del régimen laboral, ${ }^{3}$ que autoriza el pluriempleo, prohibido hasta el momento. El propósito es incrementar la producción y elevar el nivel de vida. Con ello se persigue no sólo contrarrestar el efecto económico y social del envejecimiento, sino flexibilizar el mercado laboral en gran medida precario, empobrecido e improductivo; estimular las fuerzas productivas, incrementar la productividad del trabajo, recuperar el papel del salario como estímulo de la producción material y elevar los ingresos.

La aplicación de este decreto no ha tenido el impacto esperado. Las causas podrían estar en el subempleo existente, la inadecuación del mercado de trabajo y la inexistencia de una cultura del trabajo independiente. Debe señalarse que los profesionales, investigadores, profesores, maestros, auditores, etcétera, no están autorizados para ejercer el pluriempleo. En 2009, apenas 50 mil personas practicaban el pluriempleo, $97 \%$ se contrató en los servicios — sistema educacional- siendo así improductivo. ${ }^{4}$

En 2010 se aplica otra medida. Ahora con el propósito de incrementar la productividad del trabajo por la vía de reducir el empleo superfluo. Se establece la política de reordenamiento de fuerza de trabajo, que conlleva a la reducción de aproximadamente un millón de trabajadores estatales, convirtiéndose en una de las medidas más severas de la reforma económica. ${ }^{5} \mathrm{El}$ reordenamiento laboral también busca un equilibrio en la distribución de la fuerza laboral entre los diferentes sectores de la economía. Según la Oficina Nacional de Estadística (ONE), ${ }^{6}$ la población ocupada ascendía a 5’074,400 efectivos. De ellos, $43 \%$ se encontraba empleado en sectores de servicios (presupuesto estatal, salud, educación, asistencia social), mientras 
que en sectores productivos, entre los que se encuentran la agricultura y la construcción, se destina apenas $2.4 \%$ de la población ocupada y permanecen con un alto déficit de fuerza de trabajo.

Para detener posibles repercusiones sociales de esta medida, y asimilar la fuerza de trabajo excedente, se amplía la figura del trabajo por cuenta propia (TСP). Se ampliaron hasta 181 las actividades aprobadas como alternativas privadas de empleo. A finales del 2011 la cifra de trabajadores acogidos a esta modalidad sumaban 362,920 y al cierre de mayo de 2012 esta cifra se elevaba a 387,275. ${ }^{7}$ Sin embargo, a pesar de las 181 actividades permitidas, disminuye la cantidad de actividades a las cuales se dedica la población, concentrándose en vendedores de productos agrícolas en forma ambulatoria, arrendadores de viviendas, productores-vendedores de artículos varios de uso en el hogar, mensajeros y elaboradores de alimentos - poca actividad productiva-.

Como en la sociedad cubana ha estado ausente la iniciativa privada, falta la experiencia, la capacitación en este sentido es insuficiente, las iniciativas locales son pocas y deficientes y no existe la disponibilidad de materia prima que permita actividades productivas independientes - hasta ahora no se prevé que el Estado cubano sea proveedor de ésta-. Además, las actividades aprobadas son porta- 
doras de baja productividad, escaso valor agregado y no requieren de nivel educacional.

Entre las últimas medidas se encuentra la liberación de los mercados inmobiliario y automotriz. Ambas reafirman la intensión de Raúl Castro de liberar a los cubanos de las limitaciones y prohibiciones que han tenido por décadas, y coadyuva a la ampliación de las libertades individuales dentro de la isla. La primera de estas medidas permitirá a los cubanos vender y comprar viviendas, y libera el alquiler entre nacionales, ofrece licencias para la construcción, venta de materiales, subvenciones estatales y créditos bancarios para aliviar el grave problema de la vivienda. La segunda, permite la libre importación y compra-venta de automóviles, pagando impuestos razonables y acorde con las regulaciones medioambientales. Entre las restricciones está que los autos nuevos sólo podrán comprarse en pesos convertibles cubanos o dólares, con impuesto de $4 \%$ y comprobando que el dinero fue obtenido legalmente.

Las medidas sentaron las bases para la transformación del modelo económico, dando paso a uno nuevo y reformado: el Modelo de Gestión Económica. Las tareas que debe impulsar son el aumento de la productividad y eficiencia de la economía al tiempo de mantener las conquistas sociales logradas. La política económica y social en la sección $\mathrm{VI}^{8}$ traza lineamientos generales destinados a mantener y elevar los niveles de bienestar de la población, mediante la preservación de políticas inclusivas de acceso general a la salud, educación, deporte, cultura, recreación, tranquilidad ciudadana, seguridad social y protección mediante la asistencia social a las personas que lo necesiten; el rescate del papel del trabajo y los ingresos como la vía fundamental para contribuir al desarrollo de la sociedad y la satisfacción de las necesidades personales y familiares, y, no menos importante, brindar particular atención al estudio e implementación de estrategias en todos los sectores de la sociedad para enfrentar los elevados niveles de envejecimiento de la población. ${ }^{9}$

\section{Notas}

1 Víctor M. Figueroa Albelo et al., La economía política de la construcción del socialismo, 2006, en 〈www.eumed.net/libros/2006b/vmfa〉, consultado el 10 de septiembre de 2012.

2 J. Mill, Elements of Political Economy, 1821, en 〈http://www.marxists.org/ reference/subject/economics/mill-james/ch02_2.htm>, consultado, 26 de septiembre de 2012 .

3 Gaceta oficial extraordinaria, número 022, 29/06/ 2009.

4 José A. Rodríguez, «Discreta aún la aplicación del pluriempleo en Cuba», Juventud Rebelde, 15 de diciembre, 2009, en 〈http://www.juventudrebelde. cu/cuba/2009-12-15/discreta-aun-la-aplicacion-del-pluriempleo-encuba/>, consultado el 20 de septiembre de 2012.

5 «Reordenamiento de la Fuerza de Trabajo», en «http://yohandry.wordpress.

\section{CONCLUSIÓN}

Con la caída de la uRss, Cuba quedó aislada y se enfrentó a tener que «desarrollar el socialismo en un solo país». Con el agravante de ser un país atrasado, pequeño, bloqueado económicamente y a 90 millas de Estados Unidos, y de que el cubano que llega a este último país se convierte en ciudadano por decreto. Bastó una década para que las contradicciones mostraran inviable el proyecto tal cual estaba siendo implementado. Las reformas de los últimos años no tienden a corregir el problema, sino a palear algunas inconformidades sociales (mayores libertades económicas individuales, disponibilidad de un mercado de vivienda y automóviles), a suavizar la crisis de densidad poblacional (extensión de la vida laboral) y a incrementar la productividad del empleo público (reducción de empleo superfluo). Es posible que, contra lo esperado, desaten un proceso de diferenciación social, inseguridad y violencia.

Debido al bloqueo estadounidense, es inviable un capitalismo de Estado al estilo chino, ya que nadie invertiría capitales. Latinoamérica debería defender con mayor vehemencia la sobrevivencia de esta experiencia socialista; Venezuela ya lo ha hecho con el petróleo, pero quien cuenta aquí sería Brasil. Cuba se enfrenta a pocas alternativas y muchos desafíos.

com/2010/09/17/politica-a-aplicar-en-reordenamiento-de-la-fuerza-de-trabajo-encuba/>.

6 ONE, 2010.

7 Granma Internacional, «Trabajadores por cuanta propia suman más de 385 mil», edición digital, 6 de julio, 2011, en «http://www.granma.cu/ espanol/cuba/6-julio-cuentapropistas.html, consultado el 21 de septiembre de 2012.

8 Lineamientos de la Política Económica y Social del Partido y la Revolución, 2011.

9 Ibidem. 